\title{
PERFIL E TEMPO DE PERMANÊNCIA DE PACIENTES INTENSIVOS ASSISTIDOS NA RECUPERAÇÃO PÓS-ANESTÉSICA
}

\section{Profile and length of stay of intensive care patients admitted to the post-anesthesia care unit}

\section{Perfil y estancia de pacientes intensivos asistidos en la recuperación postanestésica}

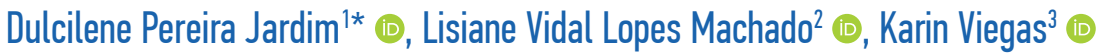

RESUMO: Objetivo: Identificar a frequência, o perfil e o tempo de permanência de pacientes intensivos admitidos na sala de recuperação pós-anestésica (SRPA). Método: Estudo transversal e retrospectivo, realizado com base em registros de admissões na SRPA de um hospital público no Rio Grande do Sul, entre julho de 2012 e julho de 2017. Resultados: No período estudado, admitiram-se no setor 22.333 pacientes, sendo 717 (3,2\%) pacientes intensivos por indisponibilidade de leito na unidade de terapia intensiva. Destes, 67,6\% eram do sexo feminino, 61,2\% em idade adulta, submetidos à neurocirurgia (61,5\%). O tempo de permanência médio no setor foi de 10,7 horas, e 4,1\% dos pacientes foram a óbito. Conclusão: A permanência de pacientes intensivos na SRPA requer adequação do setor em sua estrutura física e operacional, especialmente no que diz respeito à equipe assistencial tanto em quantidade de pessoal quanto em capacitação técnica necessária para assegurar uma assistência de qualidade.

Palavras-chave: Sala de recuperação. Período de recuperação da anestesia. Enfermagem em pós-anestésico. Enfermagem perioperatória. Cuidados críticos.

ABSTRACT: Objective: To identify the frequency, profile, and length of stay of intensive care patients admitted to the post-anesthesia care unit (PACU). Method: This is a retrospective cross-sectional study based on PACU admission records of a public hospital in Rio Grande do Sul, Southern Brazil, between July 2012 and June 2017. Results: In the study period, 22,333 patients were admitted to the PACU; 717 (3.2\%) of them were intensive care patients due to the unavailability of beds in the intensive care unit. Among them, $67.6 \%$ were women, $61.2 \%$ were adults, and $61.5 \%$ were individuals submitted to neurosurgery. The mean length of stay in the unit was 10.7 hours, and $4.1 \%$ of patients died. Conclusion: The stay of intensive care patients in the PACU requires adapting the physical and operational structure of the unit, particularly in aspects related to the care team, including the number of personnel and the technical training necessary to ensure the quality of care.

Keywords: Recovery room. Anesthesia recovery period. Postanesthesia nursing. Perioperative nursing. Critical care.

RESUMEN: Objetivo: Identificar la frecuencia, perfil y tiempo de estancia de los pacientes de cuidados intensivos ingresados en la Sala de Recuperación Posanestésica (SRPA). Método: Estudio transversal y retrospectivo, realizado a partir de los registros de ingreso en la UCPA de un hospital público de Rio Grande do Sul, entre julio de 2012 y julio de 2017. Resultados: En el período estudiado ingresaron al sector 22.333 pacientes, 717 (3,2\%) pacientes de cuidados intensivos por indisponibilidad de camas en la Unidad de Cuidados Intensivos. De estos, el 67,6\% eran mujeres, el 61,2\% adultos, sometidos a neurocirugía (61,5\%). La estancia media en el sector fue de 10,7 horas y falleció el 4,1\% de los pacientes. Conclusión: La permanencia de los pacientes de cuidados intensivos en la SRPA requiere la adecuación del sector en su estructura física y operativa, especialmente en lo que se refiere al equipo asistencial, tanto en el número de personal como en la formación técnica necesaria para asegurar una atención de calidad.

Palabras clave: Sala de recuperación. Periodo de recuperación de la anestesia. Enfermería posanestésica. Enfermería perioperatoria. Cuidados críticos.

'Mestre em Ciências pela Universidade Federal de São Paulo. Docente convidada do curso de Especialização em Enfermagem em Centro Cirúrgico, Recuperação Anestésica e Centro de Material e Esterilização da Faculdade Israelita de Ciências da Saúde Albert Einstein - São Paulo (SP) Brasil.

${ }^{2}$ Mestre em Enfermagem pela Universidade Federal de Ciências da Saúde de Porto Alegre (UFCSPA). Enfermeira no Centro Cirúrgico do Hospital Cristo Redentor/Grupo Hospitalar Conceição - Porto Alegre (RS), Brasil.

${ }^{3}$ Doutora em Gerontologia pela UFCSPA. Professora do Departamento de Enfermagem da Universidade Federal de Ciências da Saúde de Porto Alegre - Porto Alegre (RS), Brasil.

*Autora correspondente: dulcijardim@hotmail.com

Recebido: 31/05/2020 - Aprovado: 26/10/2020

https://doi.org/10.5327/Z1414-4425202000040008 


\section{INTRODUÇÃO}

A sala de recuperação pós-anestésica (SRPA) é destinada a proporcionar assistência imediata aos pacientes sob efeito de diferentes modalidades anestésicas e cirúrgicas. Para tanto, a equipe de enfermagem deve ser apropriada e treinada para prestar esse cuidado, sendo necessária, por vezes, uma assistência de alta complexidade ${ }^{1,2}$.

Nesse setor, o enfermeiro assegura a assistência até que o paciente tenha retomado a estabilidade dos seus sinais vitais, a consciência e seus reflexos protetores, considerando o procedimento cirúrgico, bem como o tipo de anestesia à qual o paciente foi submetido, além dos riscos individuais inerentes à sua história clínica pregressa ${ }^{3}$. Contudo a SRPA tem sido utilizada, na atualidade, como retaguarda da unidade de terapia intensiva (UTI), para assistência a pacientes intensivos após a realização de procedimentos cirúrgicos, em virtude da alta demanda de pacientes críticos inversamente proporcional à oferta de leitos de terapia intensiva. Outros setores hospitalares assumem esse cuidado, enquanto aguardam a liberação de leito na UTI, fazendo com que sejam horas ou até dias de permanência nessa unidade ${ }^{4}$.

A SRPA, em sua essência, presta assistência aos pacientes classificados em cuidados intermediários e semi-intensivos. Portanto admitir e assistir pacientes críticos sugere uma reflexão aprofundada sobre como garantir o cuidado com olhar clínico, levando em consideração não apenas a recuperação dos efeitos do ato anestésico-cirúrgico, mas também o cuidado integral, de forma que proporcione uma assistência que a complexidade desses pacientes exige ${ }^{5}$.

Levando em consideração que o cuidado de enfermagem deve ser precedido de planejamento, conhecer o perfil e o tempo de permanência do paciente intensivo na SRPA mostra-se de suma importância, no intuito de compreender a oferta e a demanda, preparando o setor em relação tanto aos recursos físicos e materiais necessários quanto em força de trabalho, de modo que inclua em seu quadro de colaboradores enfermeiros especialistas em UTI, a fim de garantir a qualidade da assistência aos pacientes, enquanto aguardam liberação de leito na $\mathrm{UTI}^{4,6}$.

\section{OBJETIVO}

Identificar a frequência, o perfil e o tempo de permanência de pacientes intensivos admitidos na SRPA.

\section{MÉTODO}

Estudo transversal e retrospectivo, com coleta de dados realizada em dezembro de 2017, cuja fonte das informações foram os registros de admissões de pacientes intensivos na SRPA, no período de cinco anos (julho de 2012 a julho de 2017). Coletaram-se os dados em um hospital público, com 264 leitos, referência para atendimento de pacientes politraumatizados no Rio Grande do Sul.

O centro cirúrgico (CC) dessa unidade hospitalar possui seis salas operatórias (SO) no CC central e uma SO no CC ambulatorial. São realizadas, em média, 525 cirurgias/mês, nas especialidades de neurocirurgia, traumatologia, cirurgia plástica, cirurgia vascular e cirurgia bucomaxilofacial. A SRPA é composta de 12 leitos ativos e atende pacientes não críticos em pós-operatório imediato (POI), bem como pacientes intensivos que aguardam liberação de um leito na UTI.

As variáveis para análise do estudo foram sexo, idade, especialidade cirúrgica, tempo de permanência na unidade e destino. Os dados foram organizados em planilha Excel e analisados por meio de estatística descritiva e teste de análise de variância (ANOVA), sendo considerado estatisticamente significativo $\mathrm{p}<0,05$.

Realizou-se a coleta dos dados após aprovação do projeto pelo Comitê de Ética em Pesquisa (CEP) da instituição sede do estudo, sob CAAE 78636917.8.0000.553, via Plataforma Brasil, conforme recomendações da Resolução ${ }^{\circ} 466$, de 12 de dezembro de 2012, do Conselho Nacional de Saúde ${ }^{7}$.

\section{RESULTADOS}

No período de julho de 2012 a junho de 2017, admitiram-se 22.333 pacientes na SRPA. Desses, 717 (3,2\%) eram pacientes intensivos que deveriam ter sido encaminhados para a UTI imediatamente após o procedimento anestésico-cirúrgico, mas que, por indisponibilidade de leito, foram admitidos na SRPA.

A Tabela 1 apresenta o número total de pacientes admitidos na SRPA e de pacientes intensivos, segundo mês/ano da admissão, e a Figura 1 indica a porcentagem de admissões de pacientes intensivos no setor, ao longo dos anos.

Ainda em relação à frequência, o ano de 2016 teve o maior número de admissões (14,7 pacientes/mês), e o mês de outubro foi responsável pela maior ocorrência de admissões ao longo dos anos (17,8\% pacientes/mês/ano). 
Tabela 1. Pacientes admitidos na sala de recuperação pós-anestésica e pacientes intensivos, por mês/ano (2012-2017).

\begin{tabular}{|c|c|c|c|c|c|c|c|c|c|c|c|c|}
\hline \multirow{2}{*}{ Mês } & \multicolumn{2}{|c|}{2012} & \multicolumn{2}{|c|}{2013} & \multicolumn{2}{|c|}{2014} & \multicolumn{2}{|c|}{2015} & \multicolumn{2}{|c|}{2016} & \multicolumn{2}{|c|}{2017} \\
\hline & TA & TI & TA & TI & TA & TI & TA & TI & TA & TI & TA & TI \\
\hline Jan. & - & - & 397 & 9 & 353 & 5 & 369 & 5 & 349 & 13 & 398 & 11 \\
\hline Fev. & - & - & 373 & 11 & 315 & 3 & 311 & 8 & 340 & 9 & 314 & 15 \\
\hline Mar. & - & - & 381 & 16 & 303 & 5 & 425 & 8 & 424 & 22 & 420 & 24 \\
\hline Abr. & - & - & 426 & 7 & 303 & 5 & 378 & 13 & 352 & 20 & 303 & 9 \\
\hline Maio & - & - & 470 & 18 & 358 & 5 & 381 & 15 & 388 & 14 & 418 & 8 \\
\hline Jun. & - & - & 359 & 14 & 370 & 8 & 371 & 18 & 386 & 16 & 389 & 9 \\
\hline Jul. & 377 & 03 & 381 & 24 & 403 & 6 & 401 & 23 & 334 & 9 & - & - \\
\hline Ago. & 418 & 09 & 399 & 13 & 356 & 11 & 394 & 10 & 404 & 8 & - & - \\
\hline Set. & 354 & 12 & 353 & 4 & 315 & 11 & 396 & 18 & 335 & 17 & - & - \\
\hline Out. & 413 & 13 & 374 & 18 & 362 & 13 & 375 & 18 & 345 & 27 & - & - \\
\hline Nov. & 380 & 12 & 360 & 8 & 320 & 7 & 386 & 24 & 350 & 13 & - & - \\
\hline Dez. & 380 & 9 & 369 & 6 & 386 & 10 & 398 & 9 & 391 & 9 & - & - \\
\hline Total & 2.322 & 58 & 4.642 & 148 & 4.144 & 89 & 4.585 & 169 & 4.398 & 177 & 2.242 & 76 \\
\hline
\end{tabular}

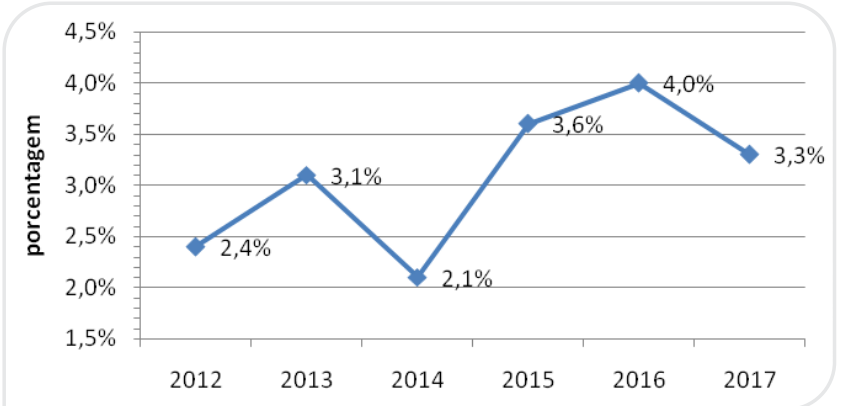

Figura 1. Porcentagem de admissões de pacientes intensivos na recuperação pós-anestésica, por ano (2012-2017).

A Tabela 2 apresenta os principais dados referentes ao perfil dos pacientes críticos internados na SRPA.

Com relação ao tempo de permanência dos pacientes intensivos em leitos na SRPA, houve variação entre 30 minutos e 237 horas, com tempo médio de 10,7 horas. A média de permanência por mês/ano está demonstrada na Tabela 3. Observou-se tendência de crescimento nos tempos médios de permanência no período investigado, sem diferença significativa entre tais tempos $(\mathrm{p}=0,510)$.

Quanto ao destino dos pacientes, 687 (95,8\%) foram transferidos para UTI após a liberação de um leito para continuidade da assistência, enquanto $30(4,1 \%)$ pacientes foram a óbito dentro da SRPA.
Tabela 2. Caracterização da amostra de pacientes intensivos na sala de recuperação pós-anestésica, segundo sexo biológico, idade e especialidade cirúrgica ( $\mathrm{n}=717)$.

\begin{tabular}{|l|c|c|}
\hline Variável & N & $\%$ \\
\hline Sexo biológico & & \\
\hline Feminino & 485 & 67,6 \\
\hline Masculino & 232 & 32,4 \\
\hline Faixa etária (anos) & & \\
\hline $4-11$ (crianças) & 6 & 0,8 \\
\hline $12-18$ (adolescentes) & 40 & 5,6 \\
\hline $19-59$ (adultos) & 439 & 61,2 \\
\hline $60-97$ (idosos) & 232 & 32,4 \\
\hline Especialidade cirúrgica & & \\
\hline Neurocirurgia & 441 & 61,5 \\
\hline Cirurgia geral & 168 & 23,4 \\
\hline Cirurgia ortopédica & 65 & 9,1 \\
\hline Cirurgia vascular & 21 & 2,9 \\
\hline Cirurgia plástica & 11 & 1,5 \\
\hline Bucomaxilofacial & 9 & 1,3 \\
\hline Cirurgia em grandes queimados & 2 & 0,3 \\
\hline Total & 717 & 100,0 \\
\hline
\end{tabular}


Tabela 3. Tempo médio de permanência do paciente intensivo na sala de recuperação pós-anestésica (em horas), por ano (2012-2017).

\begin{tabular}{|c|c|c|c|c|c|c|}
\hline Mês & 2012 & 2013 & 2014 & 2015 & 2016 & 2017 \\
\hline Janeiro & - & 11,4 & 5,8 & 11,8 & 8,3 & 11,4 \\
\hline Fevereiro & - & 15,8 & 8,6 & 6,7 & 5,4 & 17,1 \\
\hline Março & - & 13,0 & 11,4 & 7,7 & 18,9 & 15,0 \\
\hline Abril & - & 8,2 & 15,6 & 16,8 & 11,2 & 14,6 \\
\hline Maio & - & 10,5 & 6,0 & 9,0 & 14,2 & 21,2 \\
\hline Junho & - & 10,0 & 5,6 & 14,1 & 18,6 & 8,3 \\
\hline Julho & 7,6 & 13,2 & 20,8 & 15,0 & 14,3 & - \\
\hline Agosto & 10,2 & 15,0 & 8,1 & 15,6 & 5,1 & - \\
\hline Setembro & 11,0 & 7,5 & 27,5 & 12,2 & 17,5 & - \\
\hline Outubro & 11,7 & 8,2 & 21,3 & 18,1 & 24,5 & - \\
\hline Novembro & 8,2 & 14,6 & 15,5 & 12,0 & 10,6 & - \\
\hline Dezembro & 10,0 & 8,8 & 5,6 & 13,2 & 16,7 & - \\
\hline Média anual & 9,8 & 11,4 & 12,7 & 12,7 & 13,8 & 14,6 \\
\hline \multicolumn{5}{|c|}{ Tempo médio total: 10,7 horas } & & \\
\hline
\end{tabular}

\section{DISCUSSÃO}

O cenário brasileiro, no qual o déficit de leitos de UTI é considerado uma realidade, corrobora os achados desta pesquisa, que apresenta número significativo de admissões de pacientes criticamente enfermos na SRPA, com média similar ao longo dos meses e anos estudados, o que demonstra não se tratar de um evento isolado ${ }^{5,8}$.

Em relação ao perfil dos pacientes, este estudo demonstrou prevalência do sexo biológico feminino, o que difere de dados nacionais, que apontam o sexo masculino como mais acometido por causas externas e mais propenso à internação em terapia intensiva ${ }^{9-12}$. De acordo com dados do Sistema de Informação de Mortalidade (SIM) e de dados demográficos do departamento de informática do Sistema Único de Saúde (Datasus), ao sexo feminino são atribuídos os óbitos por acidentes de trânsito e por violência ${ }^{13}$.

Em relação à faixa etária dos pacientes, a maior parte encontra-se em fase adulta, na qual há mais ocorrências de acidentes e de situações ligadas à violência urbana, atribuídas, especialmente, ao comportamento agressivo e arriscado por parte dos homens, com probabilidade quase cinco vezes maior de morrerem precocemente, ou seja, para cada mulher, aproximadamente oito homens morrem no Brasil ${ }^{13}$.

Os idosos constituem o segundo grupo crítico mais assistido na SRPA, em decorrência do processo de envelhecimento associado a doenças crônico-degenerativas, bem como por resultado de acidentes domésticos, a exemplo das quedas. A assistência dispensada aos pacientes cirúrgicos idosos deve ser diferenciada, se comparada à assistência em outras faixas etárias, principalmente por questões decorrentes das mudanças do próprio processo de envelhecimento e da presença de doenças associadas que podem comprometer o equilíbrio funcional, com aumento da vulnerabilidade a complicações pós-operatórias $^{14}$.

A neurocirurgia foi a especialidade responsável pela maior parte das internações de pacientes intensivos na SRPA deste estudo, o que se justifica pelo fato de o serviço de neurocirurgia da instituição ser considerado referência estadual em alta complexidade, dispondo de leitos que correspondem a $45 \%$ dos pacientes internados no hospital. Além de realizar, em média, 900 cirurgias por ano, possui como diferencial uma relação com outros serviços especializados, proporcionando atendimento integral, prestado por 25 médicos neurocirurgiões e outros profissionais da equipe multidisciplinar ${ }^{15}$.

O tempo de permanência dos pacientes intensivos apresentou grande variação, o que coincide com o descrito em outro estudo paulista, no qual houve variação ainda mais elevada, entre 3 e 384 horas, com tempo médio de 41,4 horas ${ }^{2}$, também superior ao tempo médio apresentado nesta pesquisa e muito superior ao tempo médio de permanência de pacientes em POI ou não intensivos, que é de apenas 1,8 horas $^{8}$.

Ao se comparar a categoria de cuidados com as horas de permanência, observa-se que os pacientes em cuidados 
intensivos apresentam média de horas de permanência significativamente maior do que as demais categorias, bem como maior complexidade nos cuidados necessários ${ }^{4}$.

Assim, a admissão e a permanência de pacientes graves na SRPA causam repercussão negativa na equipe, pois o paciente intensivo, além da instabilidade das funções dos sistemas orgânicos, resultando em situações iminentes de emergência e de alerta constantes comuns aos pacientes em POI, requer, também, múltiplos procedimentos invasivos ${ }^{16}$, gerando alto nível de estresse da equipe que o atende na SRPA ${ }^{2,4,8}$.

Os pacientes críticos em leito de retaguarda requerem procedimentos que não fazem parte da rotina da SRPA, que é caracterizada por cuidados de baixa ou média complexidade. Esses procedimentos incluem a administração de dietas enterais e de medicamentos por bomba de infusão, banho de leito, prevenção de lesões por pressão, mudança de decúbito, que são realizados pela equipe de técnicos de enfermagem, na mesma proporção em que o enfermeiro desenvolve os cuidados de alta complexidade, tais como cateterismos, posicionamento para linha arterial, grandes curativos de feridas operatórias, entre outros ${ }^{2,8}$.

Nesse sentido, o dimensionamento de pessoal deve transcender questões meramente matemáticas e ser construído pela ótica qualitativa. Destaca-se, assim, a necessidade de uma sistematização flexível da assistência ${ }^{17}$, principalmente pelo fato de que a presença do paciente crítico não é constante e o tempo de permanência é oscilante, inviabilizando a confecção de uma escala engessada por parte do enfermeiro. Cabe, ainda, ressaltar a importância da presença ininterrupta do enfermeiro na SRPA, o que nem sempre é realidade em muitas instituições brasileiras ${ }^{8}$.

Com relação a esse aspecto, a Associação Brasileira de Enfermeiros em Centro Cirúrgico, Recuperação Anestésica e Centro de Material e Esterilização (SOBECC) preconiza para a SRPA a proporção de um técnico de enfermagem para cada três pacientes e um enfermeiro assistencial para cada oito leitos de pacientes não dependentes de respiradores ou três a quatro pacientes críticos ${ }^{18}$.

Ao permanecerem na SRPA, os pacientes críticos impactam diretamente a rotina do setor, sendo necessária uma adequação na quantidade de colaboradores disponíveis para a assistência, bem como no preparo técnico de tais profissionais. Há relatos de profissionais da enfermagem que se sentem despreparados para prestar assistência ao paciente crítico e requerem treinamento adequado para a função e o manuseio de equipamentos específicos. Soma-se, ainda, a possibilidade de inadequação do ambiente físico da SRPA para receber pacientes em isolamento, por exemplo, e/ ou ainda falta de recursos materiais e equipamentos que são de uso próprio da UTI, impactando a qualidade da assistência ofertada ${ }^{8}$.

Pode-se acrescentar, também, a ausência ou a demora na assistência por parte de membros da equipe multiprofissional, como terapeutas ocupacionais, fisioterapeutas e o acompanhamento $24 \mathrm{~h} /$ dia do paciente pelo médico intensivista. As dificuldades podem ser agravadas pela presença de familiares em horário destinado à visita, com aumento da circulação de pessoas no setor ${ }^{8}$.

Em relação ao destino dos pacientes graves, a grande maioria foi transferida para a UTI, mas uma parcela foi a óbito dentro da SRPA, fato que não corresponde à rotina diária do setor. A ocorrência de um óbito na SRPA reflete negativamente dois aspectos: primeiro, o abalo emocional dos colaboradores empenhados com a vida, podendo gerar neles sentimentos de fracasso ${ }^{19,20} \mathrm{e}$, segundo, em questões técnico-operacionais. Procedimentos como preparo do corpo, preenchimento de impressos, transferência para local adequado e localização da família para comunicação do ocorrido demandam tempo da equipe, que deixa de assistir os demais pacientes em $\mathrm{POI}^{4}$.

Este estudo traz limitações por ter sido desenvolvido em uma região específica do Brasil. Para tanto, ressalta-se a relevância de novos estudos sobre o tema, em diferentes regiões do país, onde a utilização da SRPA como retaguarda da UTI tem sido rotineira, para conhecimento de estratégias utilizadas por essas instituições na adequação do ambiente, dos recursos materiais e tecnológicos, bem como de adequações relacionadas à equipe de enfermagem e multiprofissional para o atendimento ao paciente crítico de forma segura e humanizada.

\section{CONCLUSÃO}

Neste estudo, foram assistidos, na SRPA, 717 pacientes críticos no período de cinco anos, com maior frequência no mês de outubro de cada ano e no ano de 2016 como um todo. Os pacientes eram, em sua maioria, mulheres, em idade adulta e submetidos a procedimentos neurocirúrgicos. O tempo de permanência médio dos pacientes na SRPA foi de 10,7 horas.

Esses resultados apontam a SRPA como uma alternativa para admitir e assistir pacientes intensivos após a realização de procedimentos cirúrgicos, dada a indisponibilidade de leitos na UTI, ainda que o setor não seja, em essência, destinado a esse tipo de atendimento. 
A assistência a pacientes críticos difere da rotina vivenciada na SRPA, cujo foco se baseia no atendimento a complicações pós-operatórias com alta rotatividade de leitos, somando-se, ainda, a falta de condições estruturais do setor para assegurar a privacidade dos pacientes em situações como óbito, por exemplo.

Esse tipo de assistência, ainda que esporádica, requer adequações no ambiente físico do setor, na quantidade e no bom funcionamento de materiais e de equipamentos, bem como na quantidade suficiente de profissionais da equipe de enfermagem e de sua adequada qualificação técnica, além da presença, em tempo integral, do enfermeiro e do médico intensivista para oferecer suporte em quaisquer situações.

A longa permanência de pacientes intensivos é uma realidade constante na instituição estudada e tem-se mostrado comum em outras realidades brasileiras, havendo necessidade de reorganização assistencial e administrativa por parte das instituições, sem sobrecarga dos profissionais envolvidos na assistência e sem prejuízos aos pacientes sob seus cuidados.

\section{REFERÊNCIAS}

1. Lima LB, Rabelo ER. Nursing workload in the post-anesthesia care unit. Acta Paul Enferm. 2013;26(2):116-22. http://doi.org/10.1590/ S0103-21002013000200003

2. Nascimento P, Jardim DP. Pacientes de cuidados intensivos em leito de retaguarda na recuperação pós-anestésica. Rev SOBECC. 2015;20(1):3844. http://doi.org/10.5327/Z1414-4425201500010005

3. Cecílio AAS, Peniche ACG, Popov DCS. Análise dos registros da pressão arterial na sala de recuperação pós-anestésica. Acta Paul Enferm. 2014;27(3):249-54. http://doi.org/10.1590/1982-0194201400042

4. Machado LVL, Jardim DP. Óbito do paciente intensivo na recuperação pós-anestésica: uma experiência descontextualizada. Rev SOBECC. 2018;23(3):130-5. https://doi.org/10.5327/Z1414-4425201800030004

5. Saraiva EL, Sousa CS. Pacientes críticos na unidade de recuperação pós-anestésica: revisão integrativa. Rev SOBECC. 2015;20(2):104-12. https://doi.org/10.5327/Z1414-4425201500020006

6. Squires, A, Jylhä V, Jun J, Ensio A, Kinnunen J. A scoping review of nursing workforce planning and forecasting research. J Nurs Manag. 2017;25(8):587-96. https://doi.org/10.1111/jonm. 12510

7. Brasil. Ministério da Saúde. Conselho Nacional de Saúde. Resolução no 466, de 12 de dezembro de 2012. Diretrizes e normas regulamentadoras de pesquisa envolvendo seres humanos. Diário Oficial da União; 2013.

8. Jardim DP, Machado LVL. Pacientes intensivos na recuperação pós-anestésica: dificuldades na assistência de enfermagem. Rev SOBECC. 2019;24(1):43-9. https://doi.org/10.5327/10.5327/ Z1414-4425201900010009

9. Brasil. Ministério daSaúde. Secretaria e Vigilância em Saúde. CoordenaçãoGeral de Informações e Análises Epidemiológicas Sistema de Informações sobre Mortalidade. Sistema de Informação sobre Mortalidade [Internet]. 2019 [acesso em 13 mar. 2019]. Disponível em: http://tabnet.datasus. gov.br/cgi/tabcgi.exe?sim/cnv/obt10uf.def

10. Nascimento MSM, Nunes EM, Medeiros RC, Souza WIM, Sousa Filho LF, Alves ESRC. Perfil epidemiológico de pacientes em unidade de terapia intensiva adulto de um hospital regional paraibano. Temas em Saúde [Internet]. 2018 [acesso em 3 maio 2019];18(1):247-65. Disponível em: http://temasemsaude.com/ wp-content/uploads/2018/04/18113.pdf
11. Castro RR, Barbosa NB, Alves T, Najberg E. Perfil das internações em unidades de terapia intensiva adulto na cidade de Anápolis - Goiás - 2012. Rev Gestão Sistemas Saúde. 2016;5(2):115-24. https://doi.org/10.5585/ rgss.v5i2.243

12. Corassa RB, Falci DM, Gontijo CF, Machado GVC, Alves PAB. Evolution of external cause mortality in Diamantina(MG), 2001 to 2012. CadSaúde Colet. 2017;25(3):302-14. https://doi.org/10.1590/1414-462X201700030258

13. Marques SHBM, Souza ACS, Vaz AA, Pelegrini AHW, Linch GFC. Mortalidade por causas externas no Brasil de 2004 A 2013. Rev Baiana Saúde Pública. 2017;41(2):394-409. https://doi.org/10.22278/23182660.2017.v41.n2.a2368

14. Giordani AT,SonobeHMS, Ezaias GM, Valério MA, BarraMR,StadlerDV.Perfil de pacientes cirúrgicos atendidos em um hospital público. Rev Enfer UFPE [lnternet]. 2015 [acesso em 6 maio 2019];9(1):54-61. Disponível em: https:// periodicos.ufpe.br/revistas/revistaenfermagem/article/view/10306/10976 https://doi.org/10.5205/reuol.6817-60679-1-ED.0901201508

15. Araújo A. Serviço de Neurocirurgia do Hospital Cristo Redentor torna-se referência nacional para atendimentos de alta complexidade. [Internet]. Grupo Hospitalar Conceição [acesso em 20 maio 2019]. Disponível em: https://www.ghc.com.br/noticia.aberta.asp?idRegistro=6883

16. Ribeiro GSR, Silva RC, Ferreira MA, Silva GR. Violações no uso de equipamentos por enfermeirosna Terapia Intensiva. Texto Contexto Enferm. 2017;26(2):e6050015. https://doi.org/10.1590/0104-07072017006050015

17. Moreno NTB, Carvalho R, Porfírio RBM. Dimensionamento de pessoal em centro cirúrgico ortopédico: real Xideal. Rev SOBECC. 2014;19(1):517. https://doi.org/10.4322/sobecc.2014.002

18. Associação Brasileira de Enfermeiros de Centro Cirúrgico, Recuperação Anestésica e Centro de Material e Esterilização. Diretrizes de práticas em enfermagem cirúrgica e processamento de produtos para a saúde. 7a ed. São Paulo: SOBECC; 2017.

19. Vicensi MC. Reflexão sobre a morte e o morrer na UTI: a perspectiva do profissional. Rev Bioética. 2016;24(1):64-72. http://doi. org/10.1590/1983-80422016241107

20. Beraldo LM, Almeida DV, Bocchi SCM. Da frustração ao enfrentamento do cuidado para a morte por técnicos de enfermagem. Rev Bras Enferm. 2015;68(6):1013-9. http://doi.org/10.1590/0034-7167.2015680603i 